\title{
ANORMALIDADES DO POTENCIAL EVOCADO VISUAL POR PADRÃO REVERSO EM PACIENTES COM ESCLEROSE MÚLTIPLA DEFINIDA
}

\author{
VITOR TUMAS", AMÉRICO CEIKI SAKAMOTO*
}

\begin{abstract}
RESUMO - O potencial evocado visual por padrão reverso, obtido por padrōes de $14^{\prime}$ e $28^{\prime}$, foi analisado retrospectivamente em 28 pacientes com diagnóstico de esclerose múltipla definida. Observamos respostas anormais em $27 / 28(96,4 \%)$ pacientes, em 31/36 (86\%) dos olhos considerados sintomáticos e em I6/20 (80\%) dos ollhos assintomáticos. Classificando os achados em cada olho segundo as respostas obtidas aos dois estímulos, observamos uma possivel relação entre essa classificação e a gravidade de comprometimento visual pela doença. Detectamos em alguns olhos anormalidades isoladas do N75 e também de P100 apenas à estimulação de 28'. Dessa forma, a técnica empregada foi considerada sensível e pôde definir inclusive comprometimento visual subclínico. Entretanto, não teve sensibilidade absoluta para detectar anormalidades em alguns olhos sintomáticos. O tipo de resposta aos estímulos empregados pode sugerir o grau de extensão de envolvimento do sistema visual pela doença. Os achados mostram ainda o envolvimento irregular e predominante das fibras mais centrais da visão pela esclerose múltipla, e sugerem processadores neurais distintos e paralelos para as respostas aos dois padrōes utilizados.
\end{abstract}

PALAVRAS-CHAVE: potencial evocado visual, esclerose múltipla.

Pattern reversal visual evoked potential abnormalities in patients with defined multiple sclerosis

SUMMARY - The pattern reversal visual evoked potential with checks of 14' and 28' was restropectivelly studied in 28 patients with definite multiple sclerosis. We observed abnormal responses in $27 / 28(96.4 \%)$ patients, in $31 / 36(86 \%)$ of symptomatic eyes, and in $16 / 20(80 \%)$ of asymptomatic eyes. When we classified the abnormalities in each eye according to the findings obtained with each check, there was a correlation between the pattern of abnormalities and the severity of visual involvement. Occasionally there were isolated abnormalities of $\mathrm{N} 75$ or only in P100 obtained with 28 ' checks. In conclusion the methodology applied was very sensible in detecting abnormalities in visual pathway. We could classify the findings in each eye and correlate them with the severity of visual involvement. The findings showed uneven distribution of lesions in visual pathway, affecting preferentially the central vision afferents.

KEY-WORDS: visual evoked potentials, multiple sclerosis

Desde que Halliday, McDonald e Mushin ${ }^{6}$ observaram anormalidades no potencial evocado visual provocadas pela neurite óptica, esse teste ficou definido como valioso especialmente para o estudo da porção pré-quiasmática da via visual em pacientes com esclerose múltipla (EM). O potencial visual evocado pela reversāo em baixa frequência de um padrão xadrez, preto e branco, é o método mais útil para a investigaçāo clínica da via visual, pela sua menor variabilidade e maior sensibilidade em detectar anormalidades ${ }^{4}$. Apesar de as anormalidades observadas no potencial evocado visual

"Pós-graduando e Médico Assistente responsável pelo Laboratório de Potenciais Evocados do Hospital das Clínicas da Faculdade de Medicina de Ribeirāo Preto da Universidade de São Paulo(FMRP/USP); "“Professor Assistente Doutor do Departamento de Neurologia, Psiquiatria e Psicologia Médica da FMRP/USP.Aceite: 12-maio-1995. 
por padrāo reverso (PEVPR) serem inespecíficas em relaçāo ao processo patológico subjacente ${ }^{3,12,16,21}$, sua principal utilidade clínica revelou-se na identificação do envolvimento subclínico da via visual em pacientes suspeitos de EM, definindo o comprometimento multifocal do sistema nervoso central (SNC) $)^{2,17}$ inclusive os critérios de Poser et al. ${ }^{20}$ para o diagnóstico da EM consideraram as anormalidades evidenciadas pelos potenciais evocados como evidência paraclínica de lesão no SNC, e destaca-se entre eles o potencial evocado visual como o mais sensível ${ }^{3,4,11,12,16,21}$.

Dessa forma, estudamos o PEVPR em pacientes com EM definida, segundo a técnica utilizada em vários laboratórios nacionais ${ }^{14,15,19}$, com a finalidade de caracterizar as anormalidades observadas nesses pacientes e correlacioná-las com a forma e a extensão do envolvimento do sistema visual pela doença.

\section{MATERIAL E MÉTODOS}

Foram analisados retrospectivamente os resultados do PEVPR de 28 pacientes que tiveram o diagnóstico de EM definida, segundo os critérios de Poser et al.$^{20}$, entre os indivíduos examinados no Laboratório de Potenciais Evocados da Seção de Neurofisiologia Clínica do Hospital das Clínicas da FMRP/USP, no período de outubro1992 a dezembro-1994. Eram 25 do sexo feminino e 3 do masculino, com idades que variaram de 19 a 62 anos (média de 36,5 anos).

Os testes foran realizados com o aparelho Nicolet Compact Four e o estimulador visual Nicolet 1015 , num ambiente em penumbra com luminância constante em todos os testes. $O$ indivíduo permanecia sentado confortavelmente, com o estimulador ao mesmo nível dos olhos e a 1 metro de distância, onde media-se sua acuidade visual. Foram testados ambos os olhos, porém o estímulo era monocular, com a oclusão do olho nāo testado. A fixação era no centro da tela e durante a sessão monitorizava-se constantemente a fixaçāo e a condição de alerta.

A tela do estimulador compreendia $14,5^{\circ} \times 10,8^{\circ}$ do campo visual e era constituida de padrão xadrez $\mathrm{cm}$ preto e branco com seus elementos individuais formando ângulos de $14^{\prime}$ ou de $28^{\prime}$ em projeção sobre os olhos. A frequência de reversão do padrão foi $1,9 / \mathrm{s}$ e iniciava-se o exame sempre pelo padrão maior (28'). Não foram medidas as luminâncias dos elementos do padrão que foram mantidas constantes em todos os testes. Entretanto, utilizamos "alto contraste" entre eles segundo o controle manual do estimulador.

As respostas foram registradas em um canal (Fpz-MO) através de eletrodos de eletrencefalograma fixos ao escalpo por pasta condutora e posicionados em Fpz (S.I. 10-20) e MO ( médio-occipital, $5 \mathrm{~cm}$ acima do ínion na linha média). Utilizou-se o eletrodo referência em Cz (S.I. 10-20). Convencionou-se que potenciais elétricos negativos em Fpz ou positivos em MO promoveriam deflexāo para cima no gráfico. As impedâncias dos eletrodos foram mantidas abaixo de $5 \mathrm{k} \Omega$ e sem diferenças significativas entre si. $O$ tempo de análise foi $250 \mathrm{~ms}$, filtro de baixa frequência de $1 \mathrm{~Hz}$ e de alta frequência de $100 \mathrm{~Hz}$. Cada promediaçāo consistiu de no mínimo 100 respostas e sempre era reproduzida antes da análise. O conversor analógico-digital com capacidade de 8 bit permitiu uma definição vertical (amplitude) de $0,086 \mathrm{WV} /$ ponto, e com 512 pontos por canal uma definição horizontal (latência) com intervalo interamostral de $0,48 \mathrm{~ms} /$ ponto.

Para cada resposta foram medidas as latências absolutas de N75 e P100, a amplitude de P100 em relação a $\mathrm{N} 75$.

Cada um dos olhos testados foi considerado como sintomático sempre que os pacientes referiam redução da acuidade visual, ou apresentavam atrofia de papila ao exame do fundo de olho, ou ainda tinham acuidade visual a I metro menor que 0,5. A intensidade de comprometimento visual foi definida comó leve, moderada ou acentuada se cada olho apresentasse respectivamente uma, duas ou três das anormalidades clínicas descritas acima.

Foram considerados testes anormais aqueles com ausência de resposta demonstrável, com prolongamento na latência de P100 e/ou com aumento na sua diferença interocular ${ }^{1}$, quando comparados aos valores de normatização próprios do laboratónio

As respostas obtidas em cada olho eram classificadas em normais ou então anormais e agrupadas para análise segundo os tipos de anormalidades de P100 observados para os estímulos de $14^{\prime}$ e 28 :

Foram analisados os tipos de respostas obtidos em cada olho com relação à intensidade clínica de comprometimento visual através do teste do qui-quadrado para tabelas de contingência, considerando a hipótese $H_{\text {II }}$ de que não havia qualquer diferença entre os tipos de respostas e a gravidade de envolvimento visual. 


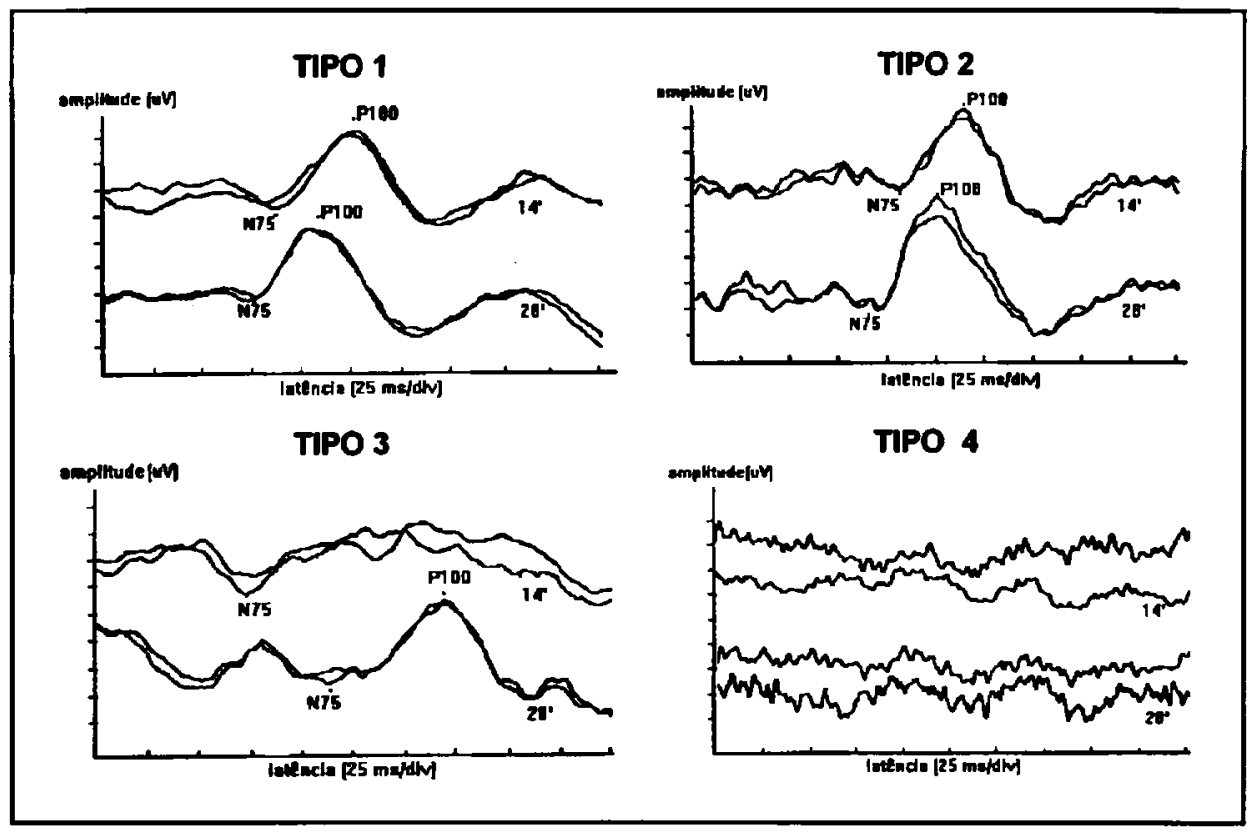

Fig. 1. Exemplos dos tipos de anormalidades observados no PEVPR dos pacientes com EM. Classificafão dos tipos no texto.

\section{RESULTADOS}

Entre os 28 pacientes estudados, $27(96,4 \%)$ tiveram anormalidades detectadas pelo PEVPR. Dos 56 olhos testados, 47 (84\%) tiveram resultados considerados anormais, enquanto 9 (16\%) nāo apresentaram qualquer anormalidade ao PEVPR. Classificamos clinicamente $36(60,7 \%)$ desses olhos como sintomáticos de qualquer intensidade, enquanto $20(39,3 \%)$ eram assintomáticos. Dos 36 olhos sintomáticos, 31 (86\%) tinham exames anormais, enquanto apenas $5(14 \%)$ tiveram o PEVPR normal. Destes últimos, 2 tinham acuidade visual (AV) $<0,5$ a 1 metro, 2 atrofia de papila ao exame do fundo de olho e 1 atrofia de papila e AV $<0,5$ a 1 metro.

Dos 20 olhos assintomáticos, 16 (80\%) tiveram o PEVPR considerado anormal, enquanto 4 (20\%) não apresentaram qualquer anormalidade ao teste.

Classificamos então os tipos de resposta observadas em cada olho (Fig 1):

TIPO 0: Resposta normal à estimulação de 14' e 28'; observada em 9 olhos $(16,1 \%)$.

TIPO 1: Aumento na latência de P100 apenas ao estímulo de 14' ou de $28^{\prime}$ : observado em 9 olhos $(16,1 \%)$.

TIPO 2: Aumento nas latências de P100 à estimulação de 28' e 14': observado em 22 olhos $(39,3 \%)$.

TIPO 3: Aumento na latência de P100 à estimulação de 28' e ausência de resposta à estimulação de 14: observado em 4 olhos $(7,1 \%)$.

TIPO 4: Ausência de resposta à estimulação de 14' e 28': observado em 12 olhos $(21,4 \%)$.

Entre os 9 olhos classificados com anormalidade do Tipo 1, apenas 3 mostraram resposta com latência de P100 prolongada à estimulação de 28 ' e resposta normal à estimulação de 14'. Esses 3 olhos eram assintomáticos. 
Tabela 1. Distribuição dos tipos de resposta observados em cada olho (0 ou 1,2, 3 ou 4), segundo a classificação definida no texto, e a intensidade de comprometimento do sistema visual definido clinicamente (assintomático, leve, moderada, severa).

\begin{tabular}{llccr}
\hline & Assintomático ou intensidade leve & Intensidade moderada & Intensidade severa & Total \\
\hline Tipos 0 ou I & $15(51 \%)$ & $2(15 \%)$ & $1(7,5 \%)$ & 18 olhos \\
Tipo 2 & $11(38 \%)$ & $10(70 \%)$ & $1(7,5 \%)$ & 22 olhos \\
Tipo 3 ou 4 & $3(11 \%)$ & $2(15 \%)$ & $11(85 \%)$ & 16 olhos \\
Total & 29 olhos & 14 olhos & 13 olhos & 56 olhos \\
& $(100 \%)$ & $(100 \%)$ & $(100 \%)$ & \\
\hline
\end{tabular}

Estabelecemos ainda a distribuição dos tipos de resposta do PEVPR em cada olho em relação à presença e intensidade de comprometimento visual, definida pela avaliação clínica como leve, moderada ou severa (Tabela 1).

O teste do Qui-quadrado para tabelas de contingência rejeitou a hipótese $H_{0}$ de que não há diferença entre as colunas ( $\leq \leq 0,001$ ), ou seja, os tipos de anormalidades são diferentes para cada intensidade de comprometimento visual. Posteriormente, a observação da tabela mostrou na intensidade assint./leve predomínio dos tipos de resposta $0 \mathrm{e} 1$, na intensidade moderada predomínio do tipo 2 e na severa dos tipos 3 e 4 . Entretanto, todos os tipos classificados de resposta do PEVPR estavam presentes em qualquer uma das condiçōes clínicas.

O componente $\mathrm{N} 75$ não foi identificado em 26 respostas à estimulaçāo por padrōes de $28^{\prime}$ e em 30 olhos à estimulação de 14'. Apesar de nāo ter sido considerado para a definição de anormalidade, em 2 olhos havia aumento discreto na sua latência com P100 normal.

A medida da diferença interocular da latência de P100 à mesma dimensāo de estímulo foi anormal em 11 pacientes e, em nenhuma vez, ocorreu como única anormalidade. Em 8 pacientes foi anormal aos estímulos de $14^{\prime}$ e $28^{\prime}$, em 1 deles ocorreu exclusivamente ao estímulo de $14^{\prime}$ e em outros 2 apenas ao estímulo de 28 '.

\section{COMENTÁRIOS}

O PEVPR detectou anormalidades no sistema visual em $27(96,4 \%)$ dos 28 pacientes estudados, confirmando a elevada incidência de envolvimento do sistema visual na EM definida. Iragui-Madoz" reviu numerosos estudos e concluiu que a frequência de anormalidades do PEV-PR em casos de EM definida varia entre $57 \%$ e $100 \%$. Essa variabilidade é decorrente da diversidade metodológica na realização dos testes e na seleção das amostras estudadas. Dos 19 estudos citados por Iragui-Madoz" 5 evidenciaram anormalidades no teste em acima de $90 \%$ dos casos. Dessa forma fica comprovado que a técnica empregada é sensível e os resultados comparáveis aos obtidos em diversos outros laboratórios.

O teste foi anormal em $47(84 \%)$ dos olhos testados e normal cm apenas $9(16 \%)$ deles. Anormalidades ocorreram em 31 (86\%) dos olhos sintomáticos e em 16 (80\%) dos assintomáticos, observando-se portanto maior incidência de anormalidades nos primeiros como era esperado ${ }^{9,11}$. A presença de respostas normais em 5 olhos sintomáticos, apesar de não terem sido excluidas outras causas de baixa acuidade visual e da arbitrariedade em definir 0,5 como limite sintomático, mostra que o método não tem sensibilidade absoluta e não pode excluir com certeza o envolvimento da via visual. Por outro lado, as anormalidades do PEVPR em olhos assintomáticos demonstram a capacidade do teste em detectar anormalidades não aparentes aos outros métodos de investigação clínica ${ }^{13}$, propriedade muito útil na investigação de pacientes com suspeita de $\mathrm{EM}^{2,8}$. Além disso, o elevado 
índice de $80 \%$ de positividade nos olhos assintomáticos deve-se provavelmente à amostra estudada de EM definida, e ao tipo de estímulo empregado que preferentemente estuda as fibras da visão mais central.

Com a classificação das respostas em cada olho em 5 tipos fundamentais, observamos relação direta entre os achados do PEVPR e a intensidade do comprometimento clínico do sistema visual, sugerindo uma relação entre essa classificação e a gravidade ou extensão da lesão no sistema visual desses pacientes. Entretanto, ficou evidente a variabilidade dos tipos de respostas do PEVPR em qualquer uma dessas condições clínicas. Por isso, consideramos então os 4 tipos de respostas anormais como participantes de um gradiente de intensidade progressiva de comprometimento visual, tal que o grupo com anormalidade mais discreta seria aquele com prolongamento de $\mathrm{P} 100$ apenas ao estímulo de 14' ou mais raramente ao de $28^{\prime}$ (Tipo 1), seguido do grupo de anormalidade moderada com latências aumentadas às duas dimensões de estímulo(Tipo 2), séguido do grupo de anormalidade acentuada com aumento da latência ao estímulo de $28^{\prime}$ e resposta ausente com 14' (Tipo 3) e, finalmente, do grupo de anormalidade severa com ausência de resposta aos dois tipos de padrão (Tipo 4).

O estímulo por padrão de 14 ' foi mais sensível que o de $28^{\prime}$ na deteç̧ão de anormalidades. Entretanto, em 3 olhos observamos respostas anormais apenas à estimulação de 28 e raramente (2 olhos) detectamos latência de N75 anormal com latência de P100 normal. Esses fatos mostram que os padrões de menores dimensōes sāo mais frequentemente anormais que os demais na EM, como concordam Hennerici et al. ${ }^{8}$, Hughes et al. ${ }^{10}$ e Novak et al. ${ }^{17}$, por serem compostos por uma frequência espacial maior e sensibilizarem os reccptores mais centrais da visāo e, segundo os últimos autores, isso concordaria com outros achados que mostram comprometimento preferencial das fibras maculares na neurite retrobulbar. Contudo, Novak et al. ${ }^{17}$ utilizando estímulos de 14', 28' e 56 ' observaram em alguns casos, como em nossos achados, anormalidade apenas no teste por 28, , o que sugere o envolvimento irregular da via visual na EM, como concordam vários outros estudos ${ }^{10.18}$, além da possibilidade de que os canais de processamento neural desses estímulos sejam diferentes. Por isso, c pelo gradiente de gravidade definido pelos tipos de respostas anormais do PEVPR, fica evidente que apesar das respostas obtidas com as duas dimensōes de estímulos poderem corresponder a canais diferentes de processamento, parece haver uma sequência paralela e próxima entre eles.

A observação de que o N75 pode ser afetado independentemente está de acordo com as observações de Ghilardi et al. ${ }^{5}$, apesar de que a sua variabilidade não nos permite definir além de uma suspeita de anormalidade ${ }^{1}$

A diferença interocular das latências de P100 ao mesmo estímulo não se mostrou tão valiosa quanto a medida da latência absoluta de P100, ao contrário do que observaram Kupersmith et al. ${ }^{13}$. Em seu estudo, entretanto, incluiram pacientes com diagnóstico possível, provável e definido de EM e enfatizaram aqueles com visāo considerada normal (20/20), enquanto o nosso limitou-se àqueles com diagnóstico exclusivo de EM definida c com acometimento mais grave da via visual. Por isso, a diferença interocular das latências provavelmente scja mais valiosa na deteç̧ão de anormalidades em olhos com comprometimento clínico mais leve. Apesar disso, não se pode desconsiderar a sua importância em definir a presença de lesāo prê-quiasmática quando as anormalidades na latência de P100 ocorrem nos dois olhos ${ }^{4}$.

\section{REFERÊNCIAS}

1. American Electroencephalographic Society. Guidelines for clinical evoked potentials studies. J Clin Neurophysiol 1984, 1:3-53.

2. Aminoff MJ. Evoked potential studies in ncurological diagnosis and management. Ann Neurol 1990, 28:706-710.

3. Chiappa KH. Pattern shift visual, brainstem auditory and short-latency somatosensory evoked potentials in multiple sclerosis. Neurology $1980,30: 110-123$. 
4. Chiappa KH. Pattem-shift visual evoked potentials: methodology. In: Chiappa K H. Evoked potentials in clinical medicine. Ed 2 New York: Raven Press, 1990, p 37-109.

5. Ghilardi MF, Sartucci F, Brannan JR, Onofrj MC, Bodis-Wollner I, Mylin L, Stroch R. N70 and P100 can be independently affected in multiple sclerosis. Electroenceph Clin Neurophysiol 1991, 80:1-7.

6. Halliday AM, McDonald WI, Mushin J. Delayed visual evoked response in optic neuritis. Lancet 1972,1:982-985.

7. Hammond SR, Yannikas C. Contribution of patttern reversal foveal and half-field stimulation to analysis of VEP abnormalities in multiple sclerosis. Electroenceph Clin Neurophysiol 1986, 64:101-118.

8. Hennerici M, Wenzel D, Freund HJ. The comparison of small-size rectangle and checkerboard stimulation for the evaluation of delayed visual evoked responses in patients suspected of multiple sclerosis. Brain 1977,100:119-136.

9. Hoeppner T, Lolas F. Visual evoked responses and visual symptoms in multiple sclerosis. J Neurol Neurosurg Psychiatry 1978, 41:493-498, .

10. Hughes JR, Stone JL, Fino JJ, Hart LA. Usefulness of different stimuli in visual evoked potentials. Neurology 1987, 37:656-662.

11. Iragui-Madoz VJ. Electrophysiology of multiple sclerosis. In Daly DD Pedley TA. Current practice of clinical electroencephalography. Ed 2 New York: Raven Press, 1990, p 707-738.

12. Kjaer $M$. Visual evoked potentials in normal subjects and patients with multiple sclerosis. Acta Neurol Scand 1980, 62:1-13.

13. Kupersmith MK, Nelson JI, Seiple WH, Carr RE, Weiss PA. The 20/20 eye in multiple sclerosis. Neurology 1983, 33:1015-1020.

14. Luccas FJC, Rodrigues-Alves CA. Potencial evocado visual por padrão reverso (PEV-PR): influência da acuidade visual. Rev Bras Oftalmol 1986, 45:97-103.

15. Luccas FJC, Manzano GM, Ragazzo PC. Potencial evocado visual por padrão reverso: estudo normativo. Arq Bras Neurocirurg 1983, 2:71-82.

16. Matthews WB, Small DG, Small M, Pountney E. Pattern reversal evoked visual potential in the diagnosis of multiple sclerosis. J Neurol Neurosurg Psychiatry 1977, 40:1009-1014.

17. Novak GP, Wiznitzer M, Kurtzberg D, Giesser BS, Vaughan HG Jr. The utility of visual evoked potentials using hemifield stimulation and several check sizes in the evaluation of suspected multiple sclerosis. Electroenceph Clin Neurophysiol 1988, 71:1-9.

18. Oishi M, Yamada T, Dickins QS, Kimura J. Visual evoked potentials by different check sizes in patients with multiple sclerosis. Neurology 1985, 35:1461-1465.

19. Oyamada MK. Contribuiçāo ao estudo de latências e de amplitude do potencial evocado visual por padrão reverso em indivíduos sadios entre 60 e 82 anos de idade.Tese de Doutorado, Faculdade de Medicina da Universidade de São Paulo, 1994.

20. Poser CM, Paty DW, Scheinberg L, McDonald WI, Davis FA, Ebers GC, Johnson KP, Sibley WA, Siiberberg DH, Tourtellote WW. New diagnostic criteria for multiple sclerosis: guidelines for research protocols. Ann Neurol 1983, 13:227-231.

21. Shahrokhi F, Chiappa KH, Young RR. Pattern shift evoked responses: two hundred patients with optic neuritis and/or multiple sclerosis. Arch Neurol 1978, 35:65-71. 